PROCEEDINGS OF THE

AMERICAN MATHEMATICAL SOCIETY

Volume 125, Number 9, September 1997, Pages 2797-2801

S 0002-9939(97)03975-0

\title{
ON THE COBORDISM INVARIANCE OF THE INDEX OF DIRAC OPERATORS
}

\author{
LIVIU I. NICOLAESCU
}

(Communicated by Palle E. T. Jorgensen)

\begin{abstract}
We describe a "tunneling" proof of the cobordism invariance of the index of Dirac operators.
\end{abstract}

The goal of this note is to present a very short proof of the cobordism invariance of the index. More precisely, if $\hat{D}$ is a Dirac operator on an odd dimensional manifold $\hat{M}$ with boundary $\partial \hat{M}=M$ then we show that the index of its restriction $D$ to $M$ is zero. The novelty of this proof consists in the fact that we provide an explicit isomorphism between the kernel and the cokernel of $D$. This map can be viewed as a sort of "propagator" (see Sect. 4).

\section{The SETTing}

Consider the following collection of data.

(a) A compact, oriented, $(2 n+1)$-dimensional Riemann manifold $\left(\hat{M}^{2 n+1}, \hat{g}\right)$ with boundary $\partial \hat{M}=M^{2 n}$ such that $\hat{g}$ is a product metric near the boundary. We denote by $s$ the longitudinal coordinate on a collar neighborhood of $M$. The various orientations are defined as in Figure 1.

(b) A bundle of complex self-adjoint Clifford modules $\hat{\mathcal{E}} \rightarrow \hat{M}$ (in the sense of $[\mathrm{BGV}])$. The Clifford multiplication is denoted by

$$
\hat{\mathbf{c}}: T^{*} \hat{M} \rightarrow \operatorname{End}(\hat{\mathcal{E}}) .
$$

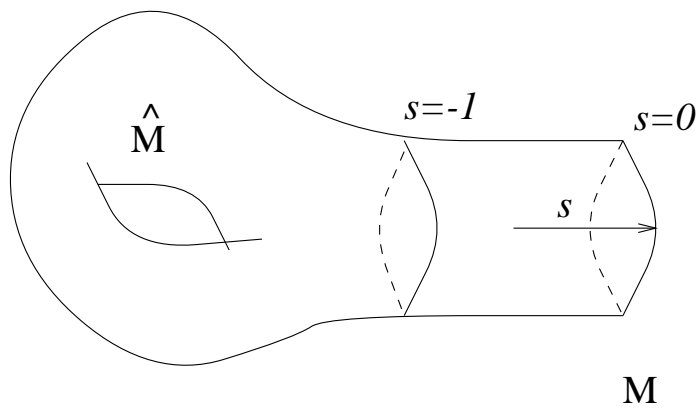

Figure 1. A $\operatorname{spin}^{c}$ bordism

Received by the editors April 22, 1996.

1991 Mathematics Subject Classification. Primary 58G10; Secondary 58G20.

(C)1997 American Mathematical Society 
Set $\mathcal{E}=\left.\hat{\mathcal{E}}\right|_{M}$. We assume that

$$
\text { End }(\mathcal{E}) \cong C l\left(T^{*} M\right) \otimes \mathbb{C},
$$

i.e. $M$ has a $\operatorname{spin}^{c}$ structure and $\mathcal{E}$ is in fact a bundle of complex spinors associated to this $\operatorname{spin}^{c}$ structure.

(c) A formally selfadjoint Dirac operator $\hat{D}: C^{\infty}(\hat{\mathcal{E}}) \rightarrow C^{\infty}(\hat{\mathcal{E}})$ such that along the neck it has the form

$$
\hat{D}=\hat{\mathbf{c}}(d s)\left(\nabla_{s}+D\right)
$$

where the operator

$$
D: C^{\infty}\left(\left.\hat{\mathcal{E}}\right|_{\{s\} \times M}\right) \rightarrow C^{\infty}\left(\left.\hat{\mathcal{E}}\right|_{\{s\} \times M}\right)
$$

is formally selfadjoint and independent of $s$. We set $J=\hat{\mathbf{c}}(d s)$. Note that since both $\hat{D}$ and $D$ are symmetric we have

$$
\{J, D\}=0
$$

where $\{\cdot, \cdot\}$ denotes the anticommutator of two operators.

Fix a local, oriented, orthonormal frame $\left(e^{1}, \cdots, e^{2 n}\right)$ of $T^{*} M$ so that $\left(d s, e^{1}, \cdots, e^{2 n}\right)$ is an oriented orthonormal frame of $T^{*} \hat{M}$. If we denote by c the Clifford multiplication along the boundary then we have the equality

$$
J \mathbf{c}\left(e^{i}\right)=\hat{\mathbf{c}}(d s) \mathbf{c}\left(e^{i}\right)=\hat{\mathbf{c}}\left(e^{i}\right)
$$

and we can conclude from (2) that $D$ is a Dirac operator on $M$ with symbol c.

We can regard $\left.J\right|_{M}$ as an endomorphism of the bundle $\mathcal{E}$ and as such it satisfies the anticommutation relations

$$
\left\{J, \mathbf{c}\left(e^{i}\right)\right\}=0 .
$$

Using (1) we conclude that $\mathbf{i} J(\mathbf{i}=\sqrt{-1})$ is a multiple of the chiral operator

$$
\Gamma_{\mathcal{E}}=\mathbf{i}^{n} \mathbf{c}\left(e^{1}\right) \cdots \mathbf{c}\left(e^{2 n}\right) .
$$

We fix the orientations such that $\mathbf{i} J=\Gamma$. Thus $\mathbf{i} J$ defines a $\mathbb{Z}_{2}$ grading on $\mathcal{E}$

$$
\mathcal{E}=\mathcal{E}_{+} \oplus \mathcal{E}_{-}, \quad \mathcal{E}_{ \pm}=\operatorname{ker}( \pm 1-\mathbf{i} J) .
$$

The anticommutation equality (3) implies that $D$ has a block decomposition

$$
D=\left[\begin{array}{lr}
0 & D_{-} \\
D_{+} & 0
\end{array}\right]
$$

where

$$
D_{ \pm}: C^{\infty}\left(\mathcal{E}_{ \pm}\right) \rightarrow C^{\infty}\left(\mathcal{E}_{\mp}\right) \text { and } D_{-}=D_{+}^{*} .
$$

Define

$$
\text { ind } D=\operatorname{dim} \operatorname{ker} D_{+}-\operatorname{dim} \operatorname{ker} D \text {. }
$$

We will show that ind $D=0$ by explicitly producing an isometry ker $D_{+} \rightarrow \operatorname{ker} D_{-}$. 


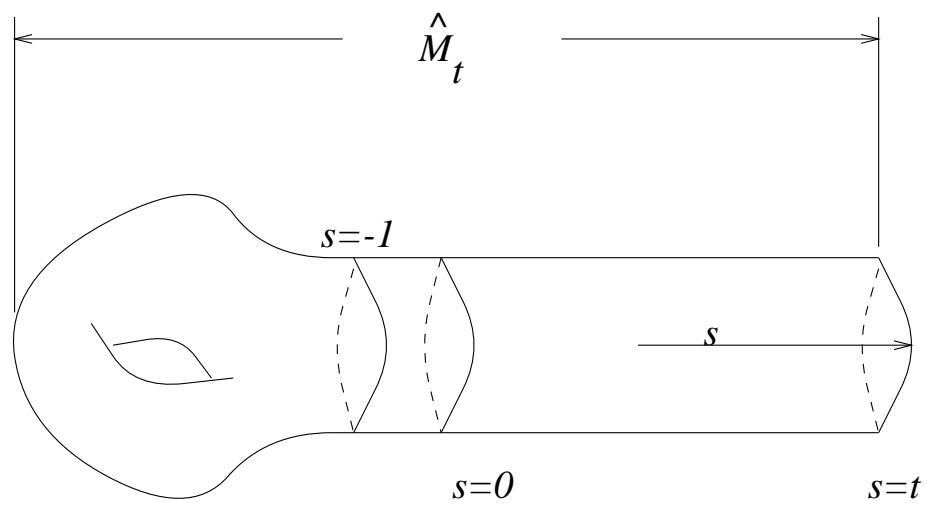

FiguRE 2. Stretching the neck

\section{CAUChY DATA SPACES AND ThEIR ADiabatiC LIMITS}

Denote by $\hat{M}_{t}(t \gg 0)$ the manifold obtained from $\hat{M}$ by attaching the long cylinder $[0, t] \times M$ (see Figure 2$)$.

The bundle $\hat{\mathcal{E}}$ and the operator $\hat{D}$ have natural extensions $\hat{\mathcal{E}}_{t}$ and $\hat{D}_{t}$ to $\hat{M}_{t}$. For every $r \geq 0$ denote by $L^{r, 2}$ the Sobolev space of distributions in $L^{2}$ with $L^{2}$ derivatives up to order $r$ and set

$$
\mathcal{K}_{t}=\left\{u \in L^{1 / 2,2}\left(\hat{\mathcal{E}}_{t}\right) ; \hat{D}_{t} u=0\right\} .
$$

In $[\mathrm{BW}]$ it is shown there exists a well defined continuous restriction map

$$
r_{t}: \mathcal{K}_{t} \rightarrow L^{2}\left(\left.\hat{\mathcal{E}}_{t}\right|_{\partial \hat{M}_{t}}\right)
$$

The Cauchy data space of $\hat{D}_{t}$ is defined as

$$
\Lambda_{t}=r_{t}\left(\mathcal{K}_{t}\right)
$$

$\Lambda_{t}$ is a closed subspace of $L^{2}(\mathcal{E})$ and satisfies a crucial condition (also established in $[\mathrm{BW}])$, namely

$$
\Lambda_{t}^{\perp}=J \Lambda_{t}
$$

The family $\left(\Lambda_{t}\right)_{t>0}$ has an especially nice behavior as $t \rightarrow \infty$. To describe it we need a bit more terminology.

For every interval $I \subset \mathbb{R}$ we denote by $\mathcal{H}_{I}$ the closed subspace of $L^{2}(\mathcal{E})$ spanned by the eigenvectors of $D$ corresponding to eigenvalues in $I$. In [N1] we proved that there exist $E \geq 0$ and a $D$ invariant subspace $L_{\infty} \subset \mathcal{H}_{[-E, E]}$ such that

$$
J L_{\infty}=L_{\infty}^{\perp}
$$

and

$$
\Lambda_{t} \stackrel{t \rightarrow \infty}{\rightarrow} \Lambda_{\infty}=L_{\infty} \oplus \mathcal{H}_{(-\infty,-E]} \text { in the gap topology of [K] }
$$

\section{The Cobordism inVariance of THE IndeX}

Denote by $P_{\infty}$ the orthogonal projection onto $\Lambda_{\infty}$ and denote by $R_{\infty}=2 P_{\infty}-1$ the orthogonal reflection in $\Lambda_{\infty}$. The condition (4) is equivalent to

$$
\left\{R_{\infty}, J\right\}=0 .
$$


Since $\mathcal{H}_{[-E, E]}$ is $J$-invariant (by (3)) we obtain a splitting

$$
\mathcal{H}_{[-E, E]}=\mathcal{H}_{E}^{+} \oplus \mathcal{H}_{E}^{-}
$$

where $\mathcal{H}_{E}^{ \pm}$is the \pm 1 -eigenspace of $\mathbf{i} J$ on $\mathcal{H}_{[-E, E]}$. The equality (6) implies that $R_{\infty}$ switches the components $\mathcal{H}_{E}^{ \pm}$, i.e.

$$
R_{\infty}\left(\mathcal{H}_{E}^{ \pm}\right)=\mathcal{H}_{E}^{\mp}
$$

This "switch" is obviously an isomorphism. Note that

$$
\text { ker } D_{ \pm} \subset \mathcal{H}_{E}^{ \pm} \text {. }
$$

Since $L_{\infty}$ is also $D$-invariant we deduce that $R_{\infty}$ maps ker $D_{+}$isometrically onto ker $D_{-}$. Geometrically this switch is the reflection in $L_{\infty}$. This shows ind $D=0$.

Remark. In [N2] we use this adiabatic limit technique to establish the cobordism invariance of the index of arbitrary families of Dirac operators. The extreme generality of that situation may obscure some nice phenomena in special cases such as the one discussed below.

\section{An EXAMPLe}

Consider a cobordism as in Figure 3 and $\hat{D}$ a Dirac operator on $\hat{M}$ with all the properties listed in Section 1 . The boundary operator $D$ consists of two pieces $D_{ \pm \infty}$ corresponding to the two components of the boundary and each is equipped with the induced chiral grading

$$
D_{ \pm \infty}=\left[\begin{array}{lr}
0 & D_{ \pm \infty}^{-} \\
D_{ \pm \infty}^{+} & 0
\end{array}\right]
$$

We assume that $\operatorname{ker} D_{ \pm \infty}^{-}=\{0\}$. Set

$$
\mathcal{H}=\mathcal{H}_{-\infty} \oplus \mathcal{H}_{\infty} \stackrel{\text { def }}{=} \operatorname{ker} D_{-\infty}^{+} \oplus \operatorname{ker} D_{\infty}^{+}
$$

Denote by $\mathcal{L}$ the space of extended $L^{2}$-solutions of $\hat{D}$ on the manifold $\hat{M}_{\infty}$ obtained by attaching infinite half-cylinders at its ends (we refer to [APS] for the exact definition). Then the space $L_{\infty} \cap \mathcal{H}$ described in Section 3 consists of the asymptotic values of these extended solutions and we can set $L_{\infty} \cap \mathcal{H}=\left.\mathcal{L}\right|_{\partial \hat{M}}$. According to the considerations in Section 3 any $\left.u \in \mathcal{L}\right|_{\partial \hat{M}}$ has an unique decomposition

$$
u=u_{+}+u_{+}, \quad u_{ \pm} \in \mathcal{H}_{ \pm \infty}
$$

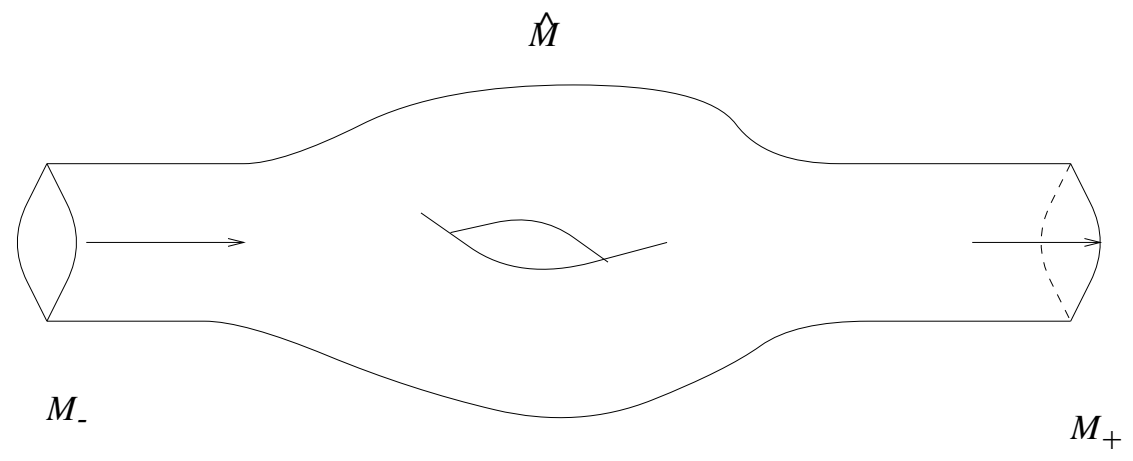

Figure 3. Cobordism 
In other words $\left.\mathcal{L}\right|_{\partial \hat{M}}$ is the graph of a linear operator (the "propagator")

$$
\mathcal{P}: u_{-} \mapsto u_{+} .
$$

The uniqueness of the decomposition (7) implies that the "propagator" $\mathcal{P}$ is an isomorphism $\mathcal{P}: \mathcal{H}_{-\infty} \rightarrow \mathcal{H}_{\infty}$.

\section{REFERENCES}

[APS] M.F. Atiyah, V.K. Patodi, I.M. Singer: Spectral asymmetry and Riemannian geometry I, Math. Proc. Cambridge Philos. Soc. 78(1975), p.405-432. MR 53:1655b

[BGV] N. Beline, E. Getzler, M. Vergne: Heat Kernels And Dirac Operators, Springer Vrelag, Berlin, 1992. MR 94e:58130

[BW] B. Boos, K. Wojciechowski: Elliptic Boundary Problems For Dirac Operators, Birckhauser, Basel, 1993. MR 94h:58168

[K] T. Kato: Perturbation Theory For Linear Operators, Springer Verlag, Berlin, 1984. MR 53:11389

[N1] L. I. Nicolaescu : The Maslov index, the spectral flow and decompositions of manifolds, Duke Math. J., vol. 80 (1995), p. 485-533. CMP 96:06

[N2] L. I. Nicolaescu: Generalized symplectic geometries and the index of families of elliptic problems, (to appear in Mem. A.M.S.) CMP 96:12

Department of Mathematics, University of Michigan, Ann Arbor, Michigan 481091109

E-mail address: liviu@math.lsa.umich.edu

Current address: Department of Mathematics, McMaster University, Hamilton, Ontario, Canada L8S 4K1 